\title{
THE LAGRANGE MULTIPLIER RULE ON MANIFOLDS AND OPTIMAL CONTROL OF NONLINEAR SYSTEMS*
}

\author{
J. C. P. BUS $\dagger$
}

\begin{abstract}
In this paper we present a differential geometric approach to the Lagrange problem and the fixed time optimal control problem for nonlinear time-invariant control systems. We restrict attention to first order conditions for optimality and present a generalized Lagrange multiplier rule for restricted variational problems. Our treatment of the optimal control problem uses a recently proposed fibre bundle approach for the definition of nonlinear systems.
\end{abstract}

Key words. Lagrange problem, nonlinear optimal control, differential geometric approach, first order conditions

1. Introduction. The classical problem of the calculus of variations is the Lagrange problem: find a curve $\phi:[0, T] \rightarrow \mathbb{R}^{n}$ from some class of curves, e.g. piecewise continuous, which satisfies certain end point conditions and minimizes an integral of the form

$$
\int_{a}^{b} \mathscr{L}(\phi(t), \dot{\phi}(t), t) d t
$$

In addition, one might impose restrictions on the curves of the form

$$
F(\phi(t), \dot{\phi}(t), t)=0 .
$$

Such problems were already studied by Euler and Lagrange at the end of the eighteenth century. A comprehensive treatment of the calculus of variations and its use to solve the (restricted) Lagrange problem is given by Carathéodory in [8]. It includes references to classical work. An important difficulty using variational techniques for solving the restricted Lagrange problem is caused by the end point conditions. It may occur that restrictions and end point conditions are such that no admissible variations of an admissible curve exist (except for the trivial one). So, such an admissible curve is extremal. Carathéodory studies this phenomenon by introducing the concept "class of the problem;" no problems arise when the class equals zero. In another general reference on the calculus of variations [3], Bliss introduces the concept "abnormality of certain order." He calls a problem normal (abnormal of order zero), if there exist nontrivial admissible variations. Both Carathéodory and Bliss need the definition and existence of Lagrange multipliers as a prerequisite for defining "class" and "normality." In this paper, which is based on unpublished course notes of Takens [20], we consider the generalized variational problem on manifolds, restricting attention to first order conditions (we speak of stationarity rather than optimality). We introduce the concept of formal stationarity for restricted problems. This is stationarity with respect to formally admissible (i.e., admissible up to first order in the variation parameter) variations. This concept is stronger than stationarity. We then define restricted variational problems to be "normal" if stationary curves are also formally stationary. Normal as we use it, means not quite the same as for Bliss. In our terminology it might occur that in normal problems there exist neither formally admissible nor admissible variations of a stationary admissible curve. It is the same for those problems which

\footnotetext{
* Received by the editors January 31, 1983, and in final revised form September 12, 1983.

† Department of Operations Research and System Theory, Mathematical Centre, Amsterdam, the Netherlands.
} 
allow nontrivial formally admissible variations. Our approach to normality does not rely upon the definition of Lagrange multipliers. The Lagrange multiplier rule is given in $\S 3$ expressing that a necessary and sufficient condition for formal stationarity for a restricted variational problem, is the existence of a stationary curve for a related unrestricted problem on a higher dimensional manifold. Then the theory of integral invariants of Cartan [7] can be used to express stationary curves for the latter problem as characteristic curves of a certain differential 2 -form. The problem of normality is postponed to $\S \S 4$ and 5 where the unrestricted Lagrange problem and the nonlinear optimal control problem are formulated as restricted variational problems. The former is merely given as an example and normality is proven, as to be expected. In our opinion the latter has value in itself. Moreover, it incorporates a recently introduced formulation of nonlinear control systems on fibre bundles (see [14], [18] and [21]). We shall see that the variational problem associated with an optimal control problem with clamped end points, will not always be normal as was already clear from the results in the books of Carathéodory and Bliss.

Variational problems on manifolds, using differential geometric concepts and Cartan's characterization for unrestricted problems, are also treated in various other papers, e.g. [10], [11], [12], [13], [15] and [17]. The restrictions considered in these references are induced by exterior differential systems or Pfaffian systems. They place more emphasis on the generalized Euler-Lagrange equation as a necessary condition for stationarity, treating the normality problem in about the same way as Bliss, except for their use of modern differential geometric results and formulations. In our approach the multiplier rule plays a natural role and normality is treated differently. Together with the linkage to the fibre bundle approach to nonlinear control systems, we expect that the given formulation of optimal control problems will be useful for studying optimal feedback control laws. It can be extended to infinite horizon problems (see [6]) in which case it might be particularly useful. The given approach is coordinate-free and does not presuppose any regularity conditions on the cost function.

Finally, in this paper we shall use the notation given in [19]. For instance, if $M$ is a smooth manifold, $T M$ is its tangent bundle ( $T_{x} M$ is the tangent space at $x \in M$ ) and $T^{*} M$ is the cotangent bundle. If $f: M \rightarrow N$ is a smooth mapping between smooth manifolds $M$ and $N$ then $f_{*}: T M \rightarrow T N$ is its lift to the tangent bundles and for any $k$-form $\omega$ on $N, f^{*} \omega$ is a $k$-form on $M$ which is defined by $\left(f^{*} \omega\right)(v)=\omega\left(f_{*} v\right)$ for all $v \in T M$. Some minor deviations from Spivak's notation occur. The set of smooth vector fields on a smooth manifold is denoted by $\mathscr{X}(M)$. Furthermore, given a $k$-form $\omega$ and a vector field $X$ on $M$, we define the contraction $\iota_{X} \omega$ of $\omega$ with respect to $X$, to be the $(k-1)$-form on $M$ defined by

for

$$
\iota_{X} \omega\left(X_{1}, \cdots, X_{k-1}\right)=\omega\left(X, X_{1}, \cdots, X_{k-1}\right)
$$

$$
X_{i} \in \mathscr{X}(M) \quad(i=1, \cdots, k-1) .
$$

Unless stated otherwise all manifolds, mappings, forms and vector fields are assumed to be smooth, i.e. $C^{\infty}$.

2. The unrestricted variational problem. Let $M$ be a smooth manifold with $\operatorname{dim} M=m, \alpha$ a smooth (differential) 1 -form on $M$ and $h: M \rightarrow \mathbb{R}$ a function. Denote $I=[0, T] \subset \mathbb{R}$. Let $x_{0} \in M$, the initial point, be given and $S \subset M$ be a connected smooth submanifold of $M$, called the target set. Define for smooth curves $\phi: I \rightarrow M$ the action

$$
\mathscr{J}(\phi)=h(\phi(T))+\int_{I} \phi^{*} \alpha .
$$


The variational problem w.r.t. this data, denoted by $\operatorname{VP}\left(M, \alpha, x_{0}, h, S\right)$ is the problem to find curves with $\phi(0)=x_{0}, \phi(T) \in S$, which are locally optimal w.r.t. $\mathscr{J}$, i.e. which produce an optimal value for $\mathscr{J}$ to small variations of the curves. We shall restrict attention to first order conditions, hence to stationarity rather than optimality.

We distinguish two cases: $h \equiv 0$,

1. Clamped end point (CE) problem. $S=\left\{m_{T}\right\}$, i.e. just one point $m_{T} \in M$, and

2. Free end point (FE) problem. $S$ a connected smooth submanifold of $M$ of dimension $\geqq 1$.

The following definitions are standard (see [10], [11] and [19]).

Definition 2.1. A mapping $\tilde{\phi}:(-\delta, \delta) \times I \rightarrow M$ (for some $\delta>0$ ) is called a variation of $\phi: I \rightarrow M$ if:

(i) $\tilde{\phi}$ is $C^{\infty}$ in each variable;

(ii) $\tilde{\phi}(0, t)=\phi(t)$ for all $t \in I$;

(iii) $\tilde{\phi}(\varepsilon, 0)=\phi(0), \tilde{\phi}(\varepsilon, T) \in S$ for all $\varepsilon \in(-\delta, \delta)$.

The set of variations of $\phi$ is denoted by $\mathscr{V}_{\phi}$ and for short we write $\phi_{\varepsilon}(t)=\tilde{\phi}(\varepsilon, t)$. Depending on $S$ we speak of CE or FE variations.

Stationary curves for the action are curves which make the first variation formula vanish. The following definition makes this precise.

Definition 2.2. A curve $\phi: I \rightarrow M$ is stationary for $\operatorname{VP}\left(M, \alpha, x_{0}, h, S\right)$ if for all $\phi_{\varepsilon} \in \mathscr{V}_{\phi}:$

$$
\left.\frac{d}{d \varepsilon}\right|_{\varepsilon=0} \mathscr{J}\left(\phi_{\varepsilon}\right)=0
$$

For given variation $\phi_{\varepsilon} \in \mathscr{V}_{\phi}$ we can choose $\bar{\phi}_{\varepsilon}$ such that $\bar{\phi}_{\varepsilon}$ is identically equal to $\phi$ in some neighbourhood of $x_{0}$ and $\int_{I}\left(\phi_{\varepsilon}^{*}-\bar{\phi}_{\varepsilon}^{*}\right) \alpha$ is arbitrarily close to zero (see [22, $\S \S 6,7]$ ). The same holds for the end point in the CE case. Therefore we may assume that variations in $\mathscr{V}_{\phi}$ are identically equal to $\phi$ in neighbourhoods of the initial point and the end point (except of course for the free directions in the FE problem).

From now on we shall assume that the curves we consider are injective immersions. This is a rather natural assumption as curves with double points are usually not optimal, because of occurrence of a loop. In such cases we can formulate the variational problem for piecewise injective curves as a sum of variational problems for each piece (see also [19]).

We can give another, equivalent, definition of stationarity in terms of vector fields along $\phi$. By a vector field along a curve $\phi: I \rightarrow M$ we mean a smooth function $V: I \rightarrow T M$ which satisfies $V(t) \in T_{\phi(t)} M$. Clearly, each variation $\tilde{\phi} \in \mathscr{V}_{\phi}$ defines a vector field $V$ along $\phi$ by the formula

$$
V(t)=\left.\frac{\partial}{\partial \varepsilon}\right|_{\varepsilon=0} \tilde{\phi}(\varepsilon, t), \quad t \in I,
$$

with

$$
V(0)=0, \quad V(T)=0(\mathrm{CE}) \text { or } V(T) \in T_{\phi(T)} S(\mathrm{FE}) .
$$

We shall denote the set of vector fields along $\phi$ satisfying (2.3) by $\mathscr{X}_{\phi}$. Conversely, given any vector field $V \in \mathscr{X}_{\phi}$, we can extend it (as $\phi$ is an injective immersion) to a vector field $X \in \mathscr{L}(M)$ and construct a variation of $\phi$ by

$$
\phi_{\varepsilon}(t)=\gamma_{X}(\varepsilon)(\phi(t))
$$


where $\gamma_{X}(\varepsilon)$ denotes the flow of $X$ over $\varepsilon$. Let now $\omega$ be an arbitrary 1-form on $M$ and let $L_{X} \omega$ denote its Lie derivative w.r.t. $X$. Then

$$
\begin{aligned}
\phi^{*} L_{X} \omega & =\phi^{*}\left(\lim _{\varepsilon \rightarrow \infty} \frac{1}{\varepsilon}\left[\left(\gamma_{X}(\varepsilon)\right)^{*} \omega-\left(\gamma_{X}(0)\right)^{*} \omega\right]\right) \\
& =\left.\frac{d}{d \varepsilon}\right|_{\varepsilon=0}\left[\left(\gamma_{X}(\varepsilon) \circ \phi\right)^{*} \omega\right]=\left.\frac{d}{d \varepsilon}\right|_{\varepsilon=0}\left(\phi_{\varepsilon}^{*} \omega\right) .
\end{aligned}
$$

We also have the well-known relation

$$
L_{X} \omega=\iota_{X} d \omega+d \iota_{X} \omega .
$$

Given $V$ along $\phi$, we have for an arbitrary smooth extension $X$ of $V$ :

$$
\phi^{*} L_{X} \omega\left(\frac{\partial}{\partial t}\right)=d \omega\left(V(t), \phi_{*}\left(\frac{\partial}{\partial t}\right)\right)+d(\omega(V(t)))\left(\frac{\partial}{\partial t}\right)=\phi^{*} L_{V} \omega\left(\frac{\partial}{\partial t}\right) .
$$

(By $\partial / \partial t$ we mean the tangent vector evaluated at $t$.) Then, for all extensions $X$ of $V$ and induced variations cf. (2.4) we have the equality

$$
\phi^{*} L_{V} \omega=\left.\frac{d}{d \varepsilon}\right|_{\varepsilon=0} \phi_{\varepsilon}^{*} \omega .
$$

So any $V \in \mathscr{X}_{\phi}$ defines a class of variations $\phi_{\varepsilon}$ of $\phi$ satisfying (2.8). These relations between vector fields along $\phi$ and variations of $\phi$ show that we can equivalently define stationarity by:

Definition $2.2^{\prime} . \quad \phi$ is stationary for $\operatorname{VP}\left(M, \alpha, x_{0}, h, S\right)$, if for all $V \in \mathscr{X}_{\phi}$ :

$$
d h(V(T))+\int_{I} \phi^{*} L_{V} \alpha=0 .
$$

This definition easily leads to a useful and well-known characterization of stationary curves.

Proposition 2.3. $\phi$ is stationary for $\operatorname{VP}\left(M, \alpha, x_{0}, h, S\right)$ if and only if

$$
\phi_{*}\left(\left.\frac{\partial}{\partial t}\right|_{t}\right) \in \operatorname{ker} d \alpha \quad \forall t \in I
$$

with $\operatorname{ker} d \alpha=\left\{v \in T M \mid d \alpha(v, w)=0, \forall w \in T_{\pi(v)} M\right\}$, and

$$
\left.(d h+\alpha)\right|_{s}(\phi(T))=0
$$

where $\left.\right|_{s}$ denotes restriction to $S$.

Proof. Using Stokes' theorem we have for $V \in \mathscr{X}_{\phi}$

$$
\int_{I} \phi^{*} L_{V} \alpha=\int_{I} \phi^{*} \iota_{V} d \alpha+\alpha(V(T))
$$

So sufficiency is trivial.

Now suppose $\phi$ is stationary and (2.10) is not satisfied for some $t \in I$. Then by the smoothness we can construct a $V$ along $\phi$ with $V(0)=0, V(T)=0$ (hence $\in T_{\phi(T)} S$ ) and

$$
\int_{I} \phi^{*} \iota_{V} d \alpha \neq 0
$$

However, this contradicts the stationarity of $\phi$. Hence (2.10), and therefore (2.11), is satisfied. 
Condition (2.10) expresses that $d \alpha$ is an integral invariant for stationary curves (see [7]). $\phi$ is called a characteristic curve of $d \theta$. Another way of looking to (2.10) is to say that $\phi$ is an integral curve of the Cartan system $C(d \alpha)$ (i.e. the Pfaffian system generated by all 1 -forms $\iota_{X} d \alpha, X \in \mathscr{X}(M)$ arbitrary) (see [11]). Condition (2.11) is the so-called transversality condition at the end point. It is interpreted as to disappear, or be trivially satisfied, in the CE problem ( $h \equiv 0$ and $S$ consists of one point).

3. The restricted variational problem. We may introduce restrictions on curves in $M$ via smooth codistributions on $M$. In $\S \S 4$ and 5 it is shown that the classical Lagrange problem and the nonlinear optimal control problem can be formulated as variational problems with such restrictions. Let $E$ be a given codistribution on $M$. Denote the variational problem $\operatorname{VP}\left(M, \alpha, x_{0}, h, S\right)$ with restriction $E$ by $\mathrm{VP}\left(M, \alpha, x_{0}, h, S, E\right)$. We call a curve $\phi: I \rightarrow M$ admissible for this problem if

$$
\phi^{*} \beta \equiv 0 \quad \forall \beta \in E \text {. }
$$

We shall assume throughout that $E$ is smooth and of fixed dimension $p$, spanned locally by 1 -forms $\beta_{1}, \cdots, \beta_{p}$. So locally (3.1) has to be satisfied for $\beta=\beta_{i}(i=1, \cdots, p)$ only. We denote the class of admissible variations of $\phi$ by

$$
\mathscr{V}_{\phi}^{E}=\left\{\xi_{\varepsilon} \in \mathscr{V}_{\phi} \mid \xi_{\varepsilon}^{*} \beta \equiv 0, \forall \beta \in E\right\}
$$

In the vector field terminology we consider the set of admissible vector fields $V \in \mathscr{X}_{\phi}$ :

$$
\mathscr{X}_{\phi}^{E}=\left\{V \in \mathscr{X}_{\phi} \mid \beta(V)=0, \forall \beta \in E\right\} .
$$

The following definition is then natural.

DEFINITION 3.1. An admissible curve $\phi: I \rightarrow M$ is stationary for $V P\left(M, \alpha, x_{0}, h, S, E\right)$ if one of the following two equivalent conditions is satisfied:

(i) $d /\left.d \varepsilon\right|_{\varepsilon=0} \mathscr{F}\left(\phi_{\varepsilon}\right)=0$, for all $\phi_{\varepsilon} \in \mathscr{V}_{\phi}^{E}$,

(ii) $d h(V(T))+\int_{I} \phi^{*} L_{V} \alpha=0$, for all $V \in \mathscr{X}_{\phi}^{E}$.

Note that this definition implies that isolated admissible curves, i.e. admissible curves for which there exist no admissible variations, are stationary. Such situations may occur as shows the following example.

Example 3.2. We consider on $M=T \mathbb{R}^{2} \times \mathbb{R}$ the restricted variational problem $\operatorname{VP}\left(M, \alpha, m_{0}, 0,\left\{m_{T}\right\}, E\right)$ with $m_{0}, m_{T} \in M$ and $E$ spanned by:

$$
\beta_{1}=d x-\sqrt{1+\dot{y}^{2}} d t, \quad \beta_{2}=d y-\dot{y} d t,
$$

where $(x, y, \dot{x}, \dot{y}, t)$ are coordinates for $T \mathbb{R}^{2} \times \mathbb{R}$. Now let $\phi:[0, T] \rightarrow T \mathbb{R}^{2} \times \mathbb{R}$, given by

$$
\phi(t)=\left(\phi_{x}, \phi_{y}, \phi_{\dot{x}}, \phi_{\dot{y}}, t\right)
$$

be admissible. Then

$$
\dot{\phi}_{x}=\sqrt{1+\phi_{\dot{y}}^{2}}, \quad \dot{\phi}_{y}=\phi_{\dot{y}} .
$$

So $\phi_{x}(t)$ is the length of the curve $\phi_{y}$ from 0 to $t$. Hence, any variation of $\phi_{y}$ with fixed end point yields a change of the $x$-coordinate of the end point. Therefore there are no nontrivial admissible variations of $\phi$.

Clearly, the situation of isolated admissible curves requires careful attention and its occurrence depends on both the restrictions and the end point conditions. The way we shall handle this difficulty is suggested by Takens [20]. First observe that admissible variations satisfy the codistribution constraints for all small $|\varepsilon|$. We may consider variations of the unrestricted problem which satisfy the restrictions to first order only. 
To do so denote

$$
\begin{aligned}
& \mathscr{W}_{\phi}^{E}=\left\{\xi_{\varepsilon} \in \mathscr{V}_{\phi}\left|\frac{d}{d \varepsilon}\right|_{\varepsilon=0} \xi_{\varepsilon}^{*} \beta=0, \forall \beta \in E\right\}, \\
& \mathscr{Y}_{\phi}^{E}=\left\{V \in \mathscr{X}_{\phi} \mid \phi^{*} L_{V} \beta=0, \forall \beta \in E\right\}
\end{aligned}
$$

and define:

Definition 3.3. An admissible curve $\phi: I \rightarrow M$ is formally stationary for $\operatorname{VP}\left(M, \alpha, x_{0}, h, S, E\right)$, if one of the conditions of Definition 3.1 is satisfied with $\mathscr{V}_{\phi}^{E}$ replaced by $\mathscr{W}_{\phi}^{E}$ and $\mathscr{X}_{\phi}^{E}$ by $\mathscr{Y}_{\phi}^{E}$.

We call elements of $\mathscr{W}_{\phi}^{E}$ formal variations of $\phi$. Note that $\mathscr{V}_{\phi}^{E} \subsetneq \mathscr{W}_{\phi}^{E}$, so that formal stationarity implies stationarity, but not necessarily the converse. Example 3.2 can be used to show that. We define

Definition 3.4. VP $\left(M, \alpha, x_{0}, h, S, E\right)$ is normal for an admissible curve $\phi$, if stationarity of $\phi$ implies formal stationarity of $\phi$.

We defer the problem of normality to $\S 5$, where it is studied for the special classes of variational problems which are of concern to us here. For historic reasons we use the terminology of [3]. However, our notion is weaker in the sense that it also allows the situation that neither formally admissible nor admissible variations exist.

Before giving the main result of this section we shall dwell some time upon the global character of the results to be obtained. In fact, a global problem can easily be broken up in finitely many local problems.

Proposition 3.5. Let $\phi: I \rightarrow M$ be an injective curve. Let $\left\{I^{\mu}\right\}\left(I^{\mu}=\left[a^{\mu}, b^{\mu}\right]\right)$ be a finite collection of closed subintervals of $I$ such that $\left\{\right.$ int $\left.I^{\mu}\right\}$ is an open covering of int $I$. Define $\phi^{\mu}=\left.\phi\right|_{I^{\mu}}$, the restriction of $\phi$ to $I^{\mu}$. Then, $\phi$ is (formally) stationary for the $\mathrm{CE}$ problem $\operatorname{VP}\left(M, \alpha, x_{0}, 0,\left\{x_{T}\right\}\right)$ (or its restricted variant) if and only if $\phi^{\mu}$ is (formally) stationary for $\operatorname{VP}\left(M, \alpha, \phi\left(a^{\mu}\right), 0,\left\{\phi\left(b^{\mu}\right)\right\}\right)$ (or restricted), for all $\mu$. Similarly for the FE problem VP $\left(M, \alpha, x_{0}, h, S\right)$ with local problems VP $\left(M, \alpha, \phi\left(a^{\mu}\right), h^{\mu}, S^{\mu}\right)$ where $h^{\mu}=0, S^{\mu}=M$ if $b^{\mu} \neq T$ and $h^{\mu}=h, S^{\mu}=S$, otherwise.

Proof. Recall that variations are identically equal to $\phi$ at some neighbourhood of the clamped begin (and end) point, according to the remark after Definition 2.2.

First consider stationarity for CE problems. If $\phi$ is stationary then any variation of a subproblem on $I^{\mu}$ can be considered to be a variation of $\phi$ on $I$ (equal to $\phi$ outside $\left.I^{\mu}\right)$. So stationarity holds for the subproblem. To prove the converse choose a partition of unity $\left\{f^{\mu}\right\}\left(f^{\mu}: I \rightarrow \mathbb{R}\right)$ and write

$$
\phi_{\varepsilon}=\phi+\eta_{\varepsilon}=\phi+\sum_{\mu} f^{\mu} \eta_{\varepsilon} .
$$

As $\phi_{\varepsilon}^{\mu}=\phi^{\mu}+f^{\mu} \eta_{\varepsilon}$ is a variation of $\phi^{\mu}$ the result follows immediately. For formal stationarity we need the additional observation that

$$
\left.\frac{d}{d \varepsilon}\right|_{\varepsilon=0}\left(\phi_{\varepsilon}^{\mu *} \beta\right)=\left.f^{\mu * \frac{d}{d \varepsilon}}\right|_{\varepsilon=0}\left(\eta^{*} \beta\right)=\left.f^{\mu * \frac{d}{d \varepsilon}}\right|_{\varepsilon=0}\left(\phi_{\varepsilon}^{*} \beta\right),
$$

so that global formal variations yields local formal variations and vice versa. In the case of an FE problem we note that a variation of a subproblem (both for $b^{\mu}=T$ or $b^{\mu} \neq T$ ) can be approximated arbitrarily close by an FE variation of $\phi$ on $I$ which equals $\phi$ outside $I^{\mu}$. Hence stationarity of $\phi$ yields stationarity of $\phi^{\mu}$. Conversely, note that if $\phi^{\mu}$ is FE stationary $\left(b^{\mu} \neq T\right)$ then $\phi^{\mu}$ is also CE stationary. Hence we can again use a partition of unity argument as above. 
After this intermezzo we return to the development of the main theorem. Let $\pi: T^{*} M \rightarrow M$ denote natural projection and recall from [11] the definition of the canonical 1 -form $\theta$ on $T^{*} M$ :

$$
\theta(\xi)(v)=\xi\left(\pi_{*}(v)\right) \quad \text { for all } \xi \in T^{*} M, v \in T_{\xi} T^{*} M .
$$

We need one more important 1-form.

DEFINITION 3.6. Let $M$ be a manifold with 1-form $\alpha$ and codistribution $E$ of fixed dimension. Let $\pi_{E}: E \rightarrow M$ denote the natural projection and let $\theta_{E}$ be the canonical 1-form on $T^{*} M$ restricted to $E$. Then the Cartan form $\theta_{\alpha}$ on $E$ associated with $\alpha$ is defined by

$$
\theta_{\alpha}=\pi_{E}^{*} \alpha+\theta_{E}
$$

Now we are ready to formulate the generalized Lagrange multiplier rule.

THEOREM 3.7. An injective curve $\phi: I \rightarrow M$ is formally stationary for $\operatorname{VP}\left(M, \alpha, x_{0}, h, S, E\right)$ if and only if there exists an injective curve $\eta: I \rightarrow E$ with $\pi_{E} \circ \eta=\phi$ which is stationary for $\operatorname{VP}\left(E, \theta_{\alpha}, e_{0}, h \circ \pi_{E}, \chi(S)\right)$, for some $e_{0} \in \pi_{E}^{-1}\left(x_{0}\right)$ and some section $\chi: M \rightarrow E$.

Proof. We first give the proof for the CE problem. Let $\eta: I \rightarrow E$ be given with $\pi_{E} \circ \eta=\phi$ and $\eta$ stationary for the problem on $E$. By Proposition 3.5 we can restrict attention to curves in a coordinate neighbourhood such that $E$ is spanned by forms $\beta_{1}, \cdots, \beta_{p}$ on this neighbourhood. Furthermore, note that an arbitrary vector field along $\eta$ yields a projected vector field along $\phi$ as $\phi$ and $\eta$ are injective immersions.

To prove that $\phi$ is formally stationary we first have to prove that $\phi$ is admissible. Therefore choose local coordinates $x$ for $M$ and let $\beta_{1}, \cdots, \beta_{p}$ be a local basis for $E$. Then we can give coordinates $(x, y)$ for $E$; that is, an element $\left(x, \sum_{i=1}^{p} y_{i} \beta_{i}(x)\right) \in E$ has coordinates $(x, y)\left(y=\left(y_{1}, \cdots, y_{p}\right)\right)$. By definition of the canonical form on $E \subset T^{*} M$ we have for $v \in T_{(x, y)} E$ :

$$
\theta_{E}(x, y)(v)=\left(\sum_{i=1}^{p} y_{i} \beta_{i}(x)\right)\left(\pi_{E} * v\right)=\sum_{i=1}^{p} y_{i}\left(\pi_{E}^{*} \beta_{i}\right)(v) .
$$

Therefore, given an arbitrary vector field $X$ on $E$,

$$
X=\sum_{i=1}^{n} X_{i} \frac{\partial}{\partial x_{i}}+\sum_{j=1}^{p} Y_{j} \frac{\partial}{\partial y_{j}}
$$

we obtain

$$
\begin{aligned}
\left(L_{X} \theta_{E}\right)(x, y) & =\sum_{i=1}^{p} L_{X}\left(y_{i} \pi_{E}^{*} \beta_{i}\right)(x, y) \\
& =\sum_{i=1}^{p} Y_{i}\left(\pi_{E}^{*} \beta_{i}\right)(x, y)+\sum_{i=1}^{p} y_{i} L_{X}\left(\pi_{E}^{*} \beta_{i}\right)(x, y) .
\end{aligned}
$$

Now let in these coordinates $\eta$ be given by

$$
\eta(t)=(\phi(t), \lambda(t))
$$

( $\phi$ and $\lambda$ are $x$ and $y$ coordinates, respectively) and define

$$
W_{i}(t)=\left.w_{i}(t) \frac{\partial}{\partial y_{i}}\right|_{\eta(t)}, \quad i=1, \cdots, p,
$$

where $w_{i}$ is arbitrary on $I$ with $w_{i}(0)=w_{i}(T)=0$. Clearly $W_{i}(i=1, \cdots, p)$ are vector 
fields along $\eta$ with projection $\pi_{E *} W_{i}=0$. Then with use of (3.11)

$$
\int_{I} \eta^{*} L_{W_{i}} \theta_{\alpha}=\int_{I} w_{i}(t) \eta^{*} \pi_{E}^{*} \beta_{i}+\sum_{i=1}^{p} \int_{I} \lambda_{i}(t) \eta^{*} L_{W_{i}}\left(\pi_{E}^{*} \beta_{i}\right) .
$$

Moreover, use of (2.7) shows that the last term equals zero. As the stationarity of $\eta$ makes the left-hand side of (3.13) equal to zero, we have

$$
0=\int_{I} w_{i}(t) \eta^{*} \pi_{E}^{*} \beta_{i}=\int_{I} w_{i}(t) \phi^{*} \beta_{i}
$$

for arbitrary $w_{i}$. This proves that $\phi^{*} \beta_{i}=0(i=1, \cdots, p)$, hence $\phi$ is admissible.

To prove the formal stationarity of $\phi$ let a vector field $V$ along $\phi$ with $V(0)=$ $V(T)=0$ be given in coordinates:

$$
V(t)=\left.\sum_{i=1}^{n} V_{i}(t) \frac{\partial}{\partial x_{i}}\right|_{\phi(t)} .
$$

Define a vector field $W$ along $\eta$ by

$$
W(t)=\left.\sum_{i=1}^{n} V_{i}(t) \frac{\partial}{\partial x_{i}}\right|_{\eta(t)}
$$

Then $\pi_{E *} W=V$ and the $\partial / \partial y_{i}$-components of $W$ are zero. So use of (3.11) yields:

$$
\begin{aligned}
\int_{I} \eta^{*} L_{W} \theta_{\alpha} & =\int_{I} \eta^{*} L_{W}\left(\pi_{E}^{*} \alpha\right)+\int_{I} \eta^{*} L_{W} \theta_{E} \\
& =\int \eta^{*} L_{W}\left(\pi_{E}^{*} \alpha\right)+\sum_{i=1}^{p} \int_{I} \lambda_{i}(t) \eta^{*} L_{W}\left(\pi_{E}^{*} \beta_{i}\right) .
\end{aligned}
$$

Moreover, use of (2.7) shows that

$$
\eta^{*} L_{W}\left(\pi_{E}^{*} \beta_{i}\right)\left(\left.\frac{\partial}{\partial t}\right|_{\eta(t)}\right)=\phi^{*} L_{V} \beta_{i}\left(\left.\frac{\partial}{\partial t}\right|_{\phi(t)}\right) .
$$

Substituting this in (3.14) yields

$$
\int_{I} \eta^{*} L_{W} \theta_{\alpha}=\int \phi^{*} L_{V} \alpha+\sum_{i=1}^{p} \int_{I} \lambda_{i}(t) \phi^{*} L_{V} \beta_{i}
$$

Stationarity of $\eta$ makes the left-hand side zero. So $\phi^{*} L_{V} \beta_{i}=0(i=1, \cdots, p)$ yields $\int_{I} \phi^{*} L_{V} \alpha=0$. This implies formal stationarity.

To prove the converse, let $\phi$ be formally stationary. Given any vector field $W$ along $\eta$ with $W(0)=W(T)=0$ we obtain, using (3.11),

$$
\int_{I} \eta^{*} L_{W} \theta_{\alpha}=\int_{I} \eta^{*} L_{W}\left(\pi_{E}^{*} \alpha\right)+\sum_{i=1}^{p} \int_{I} W_{y_{i}} \phi^{*} \beta_{i}+\sum_{i=1}^{p} \int_{I} \lambda_{i} \eta^{*} L_{W}\left(\pi_{E}^{*} \beta_{i}\right),
$$

with $W_{y_{i}}$ the $\partial / \partial y_{i}$-component of $W$. As $\phi$ is admissible $\left(\phi^{*} \beta_{i}=0\right)$ we obtain, with $V=\pi_{E *} W$ :

$$
\int_{I} \eta^{*} L_{W} \theta_{\alpha}=\int_{I} \phi^{*} L_{V} \alpha+\sum_{i=1}^{p} \int_{I} \lambda_{i} \phi^{*} L_{V} \beta_{i}
$$

Hence, we have to prove that we can find $\lambda_{i}: I \rightarrow \mathbb{R}(i=1, \cdots, p)$ such that for all 
$V \in \mathscr{X}_{\phi}$ the following equality is satisfied

$$
\int_{I} \phi^{*} L_{V} \alpha=-\sum_{i=1}^{p} \int_{I} \lambda_{i} \phi^{*} L_{V} \beta_{i}
$$

Note that we then have $\eta(t)=\sum_{i=1}^{p} \lambda_{i}(t) \beta_{i}(\phi(t))$ satisfying the conditions of the theorem. For simplicity we assume that $p=1$, i.e. $E$ is spanned by one 1 -form. We omit the subscripts for $\lambda$ and $\beta$. To find an appropriate $\lambda$ in this case define a vector field $Z$ along $\phi$ such that $\beta(Z)=1$ along $\phi$. Let

$$
\begin{aligned}
& \mathscr{F}_{1}=\left\{V \mid V \text { vector field along } \phi, \phi^{*} L_{V} \beta=0, V(0)=0\right\}, \\
& \mathscr{F}_{2}=\{V \mid V \text { vector field along } \phi, V=\psi Z, \psi(0)=0\} .
\end{aligned}
$$

Then, any vector field $V \in \mathscr{X}_{\phi}$ can be written uniquely as the sum

$$
V=V_{1}+V_{2}, \quad V_{1} \in \mathscr{F}_{1}, \quad V_{2} \in \mathscr{F}_{2} .
$$

This is shown by the following argument. Given $V$, the differential equation:

$$
\begin{aligned}
& \phi^{*} L_{\nu} \beta\left(\frac{\partial}{\partial t}\right)=\psi(t) d \beta\left(Z(t), \phi_{*}\left(\frac{\partial}{\partial t}\right)\right)+d \psi\left(\frac{\partial}{\partial t}\right), \\
& \psi(0)=0
\end{aligned}
$$

defines $\psi: I \rightarrow \mathbb{R}$ uniquely. Now define

$$
V_{2}=\psi Z ; \quad V_{1}=-\psi Z .
$$

Then we have the appropriate splitting as $V_{2} \in \mathscr{F}_{2}$ by choice and $V_{1} \in \mathscr{F}_{1}$ because (use (2.7) and (3.17))

$$
\phi^{*} L_{V_{1} \beta}\left(\frac{\partial}{\partial t}\right)=\phi^{*} L_{V} \beta\left(\frac{\partial}{\partial t}\right)-\psi(t) d \beta\left(Z(t), \phi_{*}\left(\frac{\partial}{\partial t}\right)\right)+d \psi\left(\frac{\partial}{\partial t}\right)=0 .
$$

Note that $V_{1}(T)=-V_{2}(T)=-\psi(T) Z(T)$ is not necessarily equal to zero. Now let $V$ be arbitrary with $V(0)=V(T)=0$ and $V=V_{1}+V_{2}=V_{1}+\psi Z$ its unique splitting. Then (2.7) and Stokes' theorem yield

$$
\int_{I} \phi^{*} L_{V} \alpha=\int_{I} \phi^{*} \iota_{V} d \alpha+\int_{I} d(\alpha(V))=\int_{I} \phi^{*} \iota_{V_{1}} d \alpha+\int_{I} \phi^{*} \iota_{V_{2}} d \alpha
$$

where $\phi^{*} \iota_{V} d \alpha(\partial / \partial t)=d \alpha\left(V(t), \phi_{*}(\partial / \partial t)\right)$, by definition.

If $\psi(T) \neq 0\left(V_{1}(T) \neq 0\right)$ we define a constant $C_{0}$ such that

$$
\int_{I} \phi^{*} L_{V} \alpha=\int_{I} \phi^{*} \iota_{V_{2}} d \alpha-C_{0} \psi(b)
$$

If $\psi(T)=0$ then $\int_{I} \phi^{*} L_{V} \alpha=\int_{I} \phi^{*} \iota_{V_{2}} d \alpha$ by the formal stationarity of $\phi$, so that we can choose $C_{0}$ arbitrarily and (3.18) still holds. Then define $\Psi_{1}, \Psi_{2}: I \rightarrow \mathbb{R}$ by

$$
\Psi_{1} d t=\phi^{*} \iota_{Z} d \beta, \quad \Psi_{2} d t=\phi^{*} \iota_{Z} d \alpha
$$

and $\lambda: I \rightarrow \mathbb{R}$ by

$$
\dot{\lambda}=\Psi_{2}+\Psi_{1} \lambda, \quad \lambda(T)=C_{0} .
$$

Then we have with use of (3.18)-(3.20)

$$
-\int_{I} \lambda \phi^{*} L_{V} \beta=\int_{I} \psi \Psi_{2} d t-C_{0} \psi(T)=\int_{I} \phi^{*} L_{V} \alpha .
$$


So the chosen $\lambda$ satisfies (3.16) for $p=1$. Hence $\eta$, given by $\eta(t)=\lambda(t) \beta(\phi(t))$, is stationary w.r.t. $\theta_{\alpha}$ and $\pi_{E} \eta=\phi$. For $p>1$ the proof is similar. For the FE problem there is only a slight difference where we use Stokes' theorem in the definition of $C_{0}$. Here we choose $C_{0}$ such that

$$
d h(V(T))+\int_{I} \phi^{*} L_{V} \alpha=\int_{I} \phi^{*} \iota_{V_{2}} d \alpha+C_{0} \psi(T),
$$

which is fine for $\psi(T) \neq 0$. If $\psi(T)=0$, then $V_{2}(T)=\psi(T) Z(T)=0 \in T_{\phi(T)} S$ and as $V=V_{2}+V_{1} \in T_{\phi(T)} S$ we also have $V_{1}(T) \in T_{\phi(T)} S$. Then formal stationarity with $V_{2}(T)=0$ shows that (3.21) holds for arbitrary choice of $C_{0}$ if $\psi(T)=0$. Then the proof is valid for the FE case. Note that the section $\chi$ defining the target set in the problem on $E$ is given locally by $\chi(x)=\left(x, C_{0}\right)$ with $C_{0}$ as in (3.18) or (3.21).

Note that the Lagrange multipliers are hidden in the formulation of Theorem 3.7. They appear in the coordinate representation as $\lambda_{i}(t)(i=1, \cdots, n)$. Theorem 3.7 forms the heart of this paper. It enables us to formulate the Lagrange problem and the optimal control problem as a problem of finding characteristic curves of the differential of a certain Cartan form (recall Proposition 2.3), provided the associated restricted variational problem is normal for admissible curves. The most significant examples of the use of Theorem 3.7 are the unrestricted Lagrange problem and the optimal control problem. We discuss these in the next sections.

4. The Lagrange problem. Consider a smooth manifold $Q$ (the configuration space) with $\operatorname{dim} Q=n$, together with its 1-jet manifold $J^{1}(I, Q)$ (see [11]), we should in fact write $J^{1}(\mathbb{R}, Q)$ but to express that $t$ is restricted to $I$ we use the above notation). Note that a point in $J^{1}(I, Q)$ consists of a point $t \in I$ together with a point $(q, v) \in T Q$. Thus

$$
J^{1}(I, Q) \cong T Q \times I,
$$

and moreover, given a curve $\psi: I \rightarrow Q$ there exists a naturally associated curve $\phi: I \rightarrow$ $J^{1}(I, Q)$ defined by

$$
\left.\phi(t)=\left(\psi_{*}\left(\frac{\partial}{\partial t} \mid\right)_{t}\right), t\right), \quad t \in I .
$$

We denote $\phi=\psi^{l}$. Now suppose we have been given:

$$
\mathscr{L}: J^{1}(I, Q) \rightarrow \mathbb{R}, \quad h: Q \rightarrow \mathbb{R},
$$

called the Lagrangian and the end cost, respectively. Then the (unrestricted) Lagrange problem is to find curves $\psi: I \rightarrow Q$, with $\psi(0)=q_{0}, \psi(T) \in S \subset Q$, which minimize the action

$$
\mathscr{J}(\psi)=h(\psi(T))+\int_{I} \mathscr{L}\left(\psi^{l}(t)\right) d t .
$$

We can formulate this as a variational problem on $M=J^{1}(I, Q)$ with restriction on curves in $M$ to be naturally associated cf. (4.2) with curves in $Q$. Using $[11, \S 0 . b]$, this restriction is defined by a codistribution $E$ on $M$, which is a canonical subbundle of $T^{*} M=T^{*}\left(J^{1}(I, Q)\right)$. Moreover, in local coordinates $\left(q_{1}, \cdots, q_{n}, \dot{q}_{1}, \cdots, \dot{q}_{n}, t\right)$ for $M$, this subbundle $E$ is spanned by 1 -forms:

$$
\beta_{i}=d q_{i}-\dot{q}_{i} d t, \quad i=1, \cdots, n .
$$

We shall call $E$ the canonical (restriction) codistribution of the Lagrange problem and 
the variational problem so obtained the Lagrange variational problem. We already noted that we may restrict attention to variations which are identical to $\phi$ on a neighbourhood of clamped end points. So, CE conditions for $\psi: I \rightarrow Q$ yield CE conditions for $\phi: I \rightarrow J^{1}(I, Q)$. Moreover, a target set $S \subset Q$ gives rise to a target set $T S \times\{T\}$ in $J^{1}(I, Q)$ with end cost $h \circ \pi\left(\pi: J^{1}(I, Q) \rightarrow Q\right.$ natural projection). The following result is important.

PROPOSITION 4.1. The Lagrange variational problem is a normal restricted variational problem for every admissible curve.

Proof. Given any admissible curve $\phi=\psi^{l}(\psi: I \rightarrow Q)$. We have to prove that if $\phi$ is stationary, then $\phi$ is formally stationary. We may restrict attention to vector fields $V \in \mathscr{X}_{\phi}$ which can be given in canonical coordinates by

$$
V(t)=\left.V^{q}(t) \frac{\partial}{\partial q}\right|_{\phi(t)}+\left.V^{\dot{q}}(t) \frac{\partial}{\partial \dot{q}}\right|_{\phi(t)} .
$$

Suppose such a vector field satisfies:

$$
\phi^{*} L_{v} \beta=0 \quad \forall \beta \in \operatorname{span}\left\{d q_{i}-\dot{q}_{i} d t\right\} .
$$

(Note that we may work locally, by Proposition 3.5.) We first assume that $Q$ is 1 -dimensional, so $E$ is spanned by the form $\beta=d q-\dot{q} d t$. Thus (4.7) implies, using (2.7):

$$
0=\phi^{*} L_{\nu} \beta\left(\frac{\partial}{\partial t}\right)=-d \dot{q} \wedge d t\left(V(t), \phi_{*}\left(\frac{\partial}{\partial t}\right)\right)+d\left(V^{q}(t)\right)\left(\frac{\partial}{\partial t}\right) .
$$

So

$$
\frac{d V^{q}(t)}{d t}=V^{\dot{q}}(t)
$$

Now choose $\phi_{\varepsilon}$ by

$$
\phi_{\varepsilon}(t)=\left(\psi(t)+\varepsilon V^{q}(t), \dot{\psi}(t)+\varepsilon V^{\dot{q}}(t), t\right) .
$$

Then $\phi_{\varepsilon}$ is a CE variation of $\phi$ according to Definition 2.1 with

$$
\left.\frac{\partial}{\partial \varepsilon}\right|_{\varepsilon=0} \phi_{\varepsilon}(t)=\left(V^{q}(t), V^{\dot{q}}(t), 0\right)
$$

and

$$
\phi_{\varepsilon}^{*} \beta\left(\frac{\partial}{\partial t}\right)=\dot{\psi}(t)+\varepsilon \dot{V}^{q}(t)-\left(\dot{\psi}(t)+\varepsilon V^{\dot{q}}(t)\right)=0,
$$

using (4.8). So $\phi_{\varepsilon}$ is an admissible CE variation of $\phi$, so that by stationarity and (4.9)

$$
0=\left.\frac{d}{d \varepsilon}\right|_{\varepsilon=0} \int_{I} \phi_{\varepsilon}^{*} \alpha=\int_{I} \phi^{*} L_{V} \alpha
$$

This proves the theorem for $\operatorname{dim} Q=1$. For $\operatorname{dim} Q>1$ the proof is similar.

A direct consequence of Proposition 4.1 and Theorem 3.7 is the following corollary.

COROLLARY 4.2. An injective curve $\psi: I \rightarrow Q$ is a stationary curve for the Lagrange problem, if and only if there exists an injective curve $\eta: I \rightarrow E$, with $E$ the canonical codistribution, such that $\pi_{E} \circ \eta=\psi^{l}$ and $\eta$ stationary for an unrestricted variational problem VP $\left(E, \theta_{\mathscr{L},} \psi^{l}(0), \tilde{h}, \tilde{S}\right)$ with Cartan form

$$
\theta_{\mathscr{L}}=\pi_{E}^{*}(\mathscr{L} d t)+\theta_{E}
$$


and

$$
\tilde{h}=h \circ \pi \circ \pi_{E}, \quad \tilde{S}=\chi(T S \times\{T\}),
$$

where $\pi_{E}: E \rightarrow M, \pi: M \rightarrow Q$ are natural projections, $\theta_{E}$ is the canonical 1 -form restricted to $E$ and $\chi: M \rightarrow E$ is some section.

Using Proposition 2.3 the stationary curves for the given unrestricted variational problem are characteristic curves of $d \theta_{\mathscr{L}}$ satisfying the transversality conditions. If we choose canonical coordinates $q, \dot{q}, t$ for $J^{1}(Q, I)$ and $\lambda$ for the fibres of $E(\beta \in E: \beta=$ $\sum_{i=1}^{n} \lambda_{i} \beta_{i}, \beta_{i}$ given by (4.5)), then

$$
\theta_{\mathscr{L}}=\sum_{i=1}^{n} \lambda_{i} \beta_{i}+\mathscr{L} d t
$$

Then $\eta: t \rightarrow(q(t), \dot{q}(t), \lambda(t), t)$ is a characteristic curve of $d \theta_{\mathscr{L}}$ if

$$
\frac{d}{d t} \lambda(t)=\frac{\partial \mathscr{L}}{\partial q}(q, \dot{q}, t), \quad \lambda(t)=\frac{\partial \mathscr{L}}{\partial \dot{q}}(q, \dot{q}, t), \quad \frac{d}{d t} q(t)=\dot{q}(t),
$$

with given initial and end point conditions for $q, \dot{q}$. This yields the Euler-Lagrange equation:

$$
\frac{d}{d t}\left(\frac{\partial \mathscr{L}}{\partial \dot{q}}(q, \dot{q}, t)\right)-\frac{\partial \mathscr{L}}{\partial q}(q, \dot{q}, t)=0
$$

as a necessary condition on optimal curves. The transversality condition yields

$$
\lambda_{i}(T)=\frac{\partial h}{\partial q_{i}}(q(T)), \quad i=1, \cdots, n .
$$

Remark 4.3. It is easily seen that we may also choose $\alpha=\mathscr{L} d t+\beta$ for any $\beta \in E$ in the formulation of the Lagrange variational problem. Indeed, we then also have $\phi^{*} \alpha=\phi^{*}(\mathscr{L} d t)$, for all admissible $\phi$. Such a choice does not change the solution of the Lagrange problem but only induces a translation of the canonical coordinates $\lambda$ in $E$.

5. The nonlinear optimal control problem. We shall first recall the notion of a general nonlinear control system as given in [4] and [21] and worked out in [18].

Definition 5.1. A nonlinear (time-invariant) control system $\Sigma=\Sigma(Q, B, f)$ is defined by a smooth manifold $Q$, a fibre bundle $\tau: B \rightarrow Q$ and a smooth map $f: B \rightarrow T Q$ such that the following diagram commutes

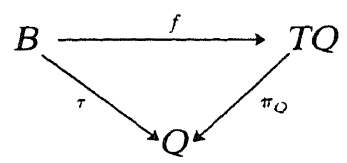

We call $\Sigma$ affine if $B$ is a vector bundle and $f$ restricted to the fibres of $B$ is an affine map into the fibres of $T Q$.

$\Sigma$ is called analytic if $B$ and $Q$ are analytic manifolds and $f$ is an analytic map.

We say that $\psi: I \rightarrow Q$ is a trajectory of $\Sigma$ if $\psi$ is absolutely continuous and

$$
\psi_{*}\left(\left.\frac{\partial}{\partial t}\right|_{t}\right) \in f\left(\tau^{-1}(\psi(t))\right)
$$

almost everywhere on $I$. With each trajectory $\psi$ we can associate a state-input trajectory 
$\zeta: I \rightarrow B$ such that

$$
\tau(\zeta(t))=\psi(t), \quad \psi_{*}\left(\left.\frac{\partial}{\partial t}\right|_{t}\right)=f(\zeta(t)), \quad t \in I .
$$

$Q$ is called the configuration space cf. the Lagrange context. The fibres of $B$ represent the (state dependent) input spaces. In local coordinates $q$ for $Q$ and $u$ for the fibres $\tau^{-1}(q)$ we obtain the familiar equation $\dot{q}=f(q, u)$ (with abuse of notation $f:(q, u) \rightarrow(q, f(q, u)))$. A state-input trajectory $\zeta$ will in such coordinates be denoted by: $\zeta(t)=(\psi(t), \nu(t)), \psi$ and $\nu$ denoting the $q$ and $u$ coordinates respectively. In the sequel we will use $f$ in both ways, how it is used will be clear from the context. If $\Sigma$ is affine then, in coordinates, $f$ has the form

$$
f(q, u)=f_{0}(q)+\sum_{i=1}^{m} u_{i} f_{i}(q),
$$

with $u_{i} \in \mathbb{R}, f_{0}$ and $f_{i}$ vector fields on $Q(i=1, \cdots, m)$.

We shall assume in the rest of this paper that $f$ is an injective immersion.

Now, an optimal control problem can be interpreted as a certain variational problem on the space of states and inputs, i.e. $B$, under certain restrictions, one of these being the restriction to curves in $B$ which are state-input trajectories of the system. In fact, the approach to the Lagrangian problem for curves in $Q$ can be followed here with respect to curves in $B$. Therefore, let us first assume to be given a function $\mathscr{G}: J^{1}(I, B) \rightarrow \mathbb{R}$, in analogy with the Lagrangian $\mathscr{L}$ in $\S 4$ and an end cost function $h: Q \rightarrow \mathbb{R}$. We restrict attention to two cases:

$\mathrm{CE}$ optimal control problem: $h \equiv 0$, clamped end point;

FE optimal control problem: $S=Q$.

The optimal control problem $\mathrm{OP}\left(\Sigma, \mathscr{G}_{,}, q_{0}, h\right)$ is to find $\zeta: I \rightarrow B$ of $\Sigma$ with $\tau \circ \zeta(0)=$ $q_{0}, \tau \circ \zeta(T) \in S$ and which are optimal w.r.t. the action

$$
\mathscr{J}(\zeta)=h(\tau \circ \zeta(T))+\int_{I} \mathscr{G}\left(\zeta^{l}(t)\right) d t .
$$

As before we restrict attention to stationarity rather than optimality. The optimal control problem can be defined as a variational problem on $J^{1}(I, B)$ where the curves are restricted to be naturally associated (cf. (4.2)) with curves in $B$ which are state-input trajectories of $\Sigma$. This implies restriction to a submanifold $M \subset J^{1}(I, B)$ defined by

$$
M=\left\{(w, t) \in J^{1}(I, B) \mid f \circ \pi(w, t)=\tau_{*}(w)\right\},
$$

with $\pi: J^{1}(I, B) \rightarrow B$ natural projection, together with restriction to the canonical restriction codistribution on $J^{1}(I, B)$, similar as in the Lagrange problem. Therefore the given optimal control problem can be defined as a restricted variational problem VP $\left(M, \alpha, x_{0}, \tilde{h}, \tilde{S}, E\right)$, with $E$ the canonical codistribution on $J^{1}(I, B)$ restricted to $M, \alpha=\left.\mathscr{G}\right|_{M} d t, \tau \circ \pi\left(x_{0}\right)=q_{0}$ and $\tilde{h} \equiv 0, \tilde{S}$ one point in the CE case, or $\tilde{h}=\tau \circ \pi \circ h$ and $\tilde{S}=(\mathrm{TQ} \times\{T\}) \cap M$ in the FE case. If we choose local coordinates $q$ on $Q, u$ on the fibres $\tau^{-1}(q)$, then canonical coordinates on $J^{1}(I, B)$ are given by $(q, u, \dot{q}, \dot{u}, t)$. Elements of $M$ are then given by $(q, u, f(q, u), \dot{u}, t)$, so that as $f$ is an injective immersion, natural coordinates on $M$ are given by $(q, u, \dot{u}, t)$. Then $E$, the canonical codistribution restricted to $M$, is locally spanned by the 1 -forms

$$
\begin{array}{ll}
\beta_{i}=d q_{i}+f_{i}(q, u) d t, & i=1, \cdots, n, \\
\beta_{n+j}=d u_{j}-\dot{u}_{j} d t, & j=1, \cdots, m,
\end{array}
$$

where $f_{i}(q, u)$ denotes the $i$ th coordinate of $f(q, u) \in T_{q} Q$. 
The question arises whether this variational problem is normal. The answer to this question appears to be relatively easy for the important class of affine analytic control systems, if we use some recent geometric techniques (see e.g. [14] and [15]). Let the system be given by $(5.3)$, let $\mathfrak{R}(\Sigma)$ denote the Lie algebra generated by $f_{i}(i=0,1, \cdots, m)$ and $\Im\left(q_{0}\right)$ the maximal integral submanifold of $\mathfrak{L}(\Sigma)$ containing the trajectory under consideration which initiates at $q_{0}$. Define $\operatorname{ad}^{0}\left(f_{0}, f_{i}\right)=f_{i}$, $\operatorname{ad}^{k+1}\left(f_{0}, f_{i}\right)=\left[f_{0}, \operatorname{ad}^{k}\left(f_{0}, f_{i}\right)\right]$ for $k=0,1, \cdots$, and $\mathfrak{S}^{1}=\left\{\operatorname{ad}^{k}\left(f_{0}, f_{i}\right) ; k=0,1, \cdots, i=\right.$ $1, \cdots, m\}$. Then we can give the following proposition.

Proposition 5.2. Let $\Sigma$ be analytic and affine. Then:

The $\mathrm{FE}$ variational problems associated with $\mathrm{OP}\left(\Sigma, \mathscr{G}, q_{0}, h, Q\right)$ are normal.

If rank $\Im^{1}\left(q_{0}\right)=\operatorname{dim} \Im\left(q_{0}\right)$, then the $\mathrm{CE}$ variational problems associated with OP $\left(\Sigma, \mathscr{G}, q_{0}, 0,\left\{q_{1}\right\}\right)$ are normal.

Remark 5.3. The condition in the $\mathrm{CE}$ case implies that the system restricted to $\Im\left(q_{0}\right)$ has a controllable linear variational equation along the trajectory initiating at $q_{0}$, or this restricted system is locally controllable of first order along this trajectory (see [2], [15] and [16]).

Proof. We assume that $\Im\left(q_{0}\right)=n$. The other cases are proved similarly by restricting the system to the lower dimensional manifold $\Im\left(q_{0}\right)$. The manifold appearing in the variational problem may be given coordinates such that $\phi(t)=\left(\phi^{q}(t), 0,0, t\right)$ (trajectory $\phi^{q}$ in $Q$ for input $u \equiv 0$ ). By breaking up the global problem in a series of local problems we may assume that $\phi(t)$ belongs to this coordinate neighbourhood for all $t \in I$. Let a formal variation in these coordinates be given by

$$
\xi(\varepsilon, t)=\left(\xi^{q}(\varepsilon, t), \xi^{u}(\varepsilon, t), \xi^{\dot{u}}(\varepsilon, t), t\right) .
$$

We shall prove that under the given conditions we can find an admissible variation $\bar{\xi}_{\varepsilon}$ of $\phi$ which is an order $\varepsilon^{2}$ perturbation of $\xi$. As stationarity involves first order conditions in $\varepsilon$ only, the proof then follows immediately. Working out the conditions for formal variations we see that

$$
\begin{aligned}
& \xi^{q}(\varepsilon, t)=\phi^{q}(t)+\varepsilon \eta(t)+\bar{C}^{q}(\varepsilon, t), \\
& \xi^{u}(\varepsilon, t)=\varepsilon \mu(t)+\bar{C}^{u}(\varepsilon, t), \\
& \xi^{\dot{u}}(\varepsilon, t)=\varepsilon \dot{\mu}(t)+\bar{C}^{\dot{u}}(\varepsilon, t),
\end{aligned}
$$

where $\bar{C}^{q}(\varepsilon, t), \bar{C}^{u}(\varepsilon, t)$ and $\bar{C}^{\dot{u}}(\varepsilon, t)$ are all of order $\varepsilon^{2}$ and equal to zero for $t=0$ and arbitrary $\varepsilon$. Moreover, $\eta(t)$ satisfies the linear equation of variations with input $\mu(t)=\left(\mu_{1}(t), \cdots, \mu_{m}(t)\right)^{T}$ :

$$
\begin{aligned}
& \dot{\eta}(t)=\frac{d f_{0}}{d q}\left(\phi^{q}(t)\right) \cdot \eta(t)+\sum_{i=1}^{m} f_{i}\left(\phi^{q}(t)\right) \cdot \mu_{i}(t), \\
& \eta(0)=0 .
\end{aligned}
$$

Now consider for arbitrary $C^{u}(\varepsilon, t)=\left(C_{1}^{u}(\varepsilon, t), \cdots, C_{m}^{u}(\varepsilon, t)\right)^{T}$ of order $\varepsilon^{2}$ and satisfying $C^{u}(\varepsilon, 0)=\dot{C}^{u}(\varepsilon, 0)=0($ arbitrary $\varepsilon)$ the equation

$$
\begin{aligned}
& \dot{q}(t)=f_{0}(q(t))+\sum_{i=1}^{m} f_{i}(q(t))\left(\varepsilon \mu_{i}(t)+C_{i}^{u}(\varepsilon, t)\right), \\
& q(0)=q_{0} .
\end{aligned}
$$

Then from [5, Thm. 6] and [9], we know that for $\varepsilon$ small enough any solution of (5.17) can be written as a unique convergent Volterra series. Working out this series and 
using the facts that $\phi^{q}$ is a trajectory of (5.10) for $\mu=0$ and $\eta$ satisfies (5.9), we see that any solution of $(5.10)$ can be written as $\zeta^{q}(\varepsilon, t)=\phi^{q}(t)+\varepsilon \eta(t)+C^{q}(\varepsilon, t)$, with $C^{q}(\varepsilon, 0)=0, C^{q}(\varepsilon, t)=O\left(\varepsilon^{2}\right)$. Hence,

$$
\zeta^{q}(\varepsilon, t)=\left(\zeta^{q}(\varepsilon, t), \varepsilon \mu(t)+C^{u}(\varepsilon, t), \varepsilon \dot{u}(t)+\dot{C}^{u}(\varepsilon, t), t\right)
$$

is an admissible variation and an $\varepsilon^{2}$ perturbation of a formal variation. This proves the assertion for $\mathrm{FE}$ problems. For CE problems we still have to show that we can choose $C^{u}(\varepsilon, t)$ such that the solution $\zeta^{q}(\varepsilon, t)$ satisfies $\zeta^{q}(\varepsilon, 1)=q_{1}=\phi^{q}(1)$ (i.e. $\left.C^{q}(\varepsilon, 1)=0\right)$. By the local controllability of the system we can find $\tilde{C}^{u}(\varepsilon, t)$ such that $\varepsilon \mu_{i}(t)+C^{u}(\varepsilon, t)+\tilde{C}^{u}(\varepsilon, t)$ yields a trajectory of (5.10) terminating in $q_{1}$. Moreover, the fact that the linear equation of variations $(5.9)$ is controllable assures that we can choose $\tilde{C}^{u}(\varepsilon, t)$ of the same order in $\varepsilon$ as $C^{q}(\varepsilon, 1)$, i.e. $O\left(\varepsilon^{2}\right)$. This completes the proof of the proposition.

The restriction to affine systems does not seem to be essential. The results of Brockett and Crouch yielding the Volterra series solution for (5.10) can also be given for nonaffine systems.

As controllable linear systems are first order controllable, the condition rank $\varsigma^{1}\left(q_{0}\right)=\operatorname{dim} \Im\left(q_{0}\right)$ is satisfied for all (also noncontrollable) linear systems.

We give one example of a system which is controllable but not first order locally controllable and which may give nonnormal variational problems.

Example 5.4. Let $I=[0,1], q^{0}=(0,0)^{T}$ and

$$
\dot{q}_{1}=u, \quad \dot{q}_{2}=q_{1}^{2}+1
$$

Then

$$
f_{0}=\left(\begin{array}{c}
0 \\
q_{1}^{2}+1
\end{array}\right), \quad f_{1}=\left(\begin{array}{l}
1 \\
0
\end{array}\right), \quad\left[f_{0}, f_{1}\right]=\left(\begin{array}{c}
0 \\
2 q_{1}
\end{array}\right), \quad \operatorname{ad}^{k}\left(f_{0}, f_{1}\right)=0 \quad \text { for } k \geqq 2 .
$$

However

$$
\left[f_{1},\left[f_{0}, f_{1}\right]\right]=-\left(\begin{array}{l}
0 \\
2
\end{array}\right)
$$

so that the system is locally controllable, but

$$
\mathbb{S}^{1}\left(q_{0}\right)=\operatorname{span}\left\{\left(\begin{array}{l}
1 \\
0
\end{array}\right)\right\}
$$

For $u=0$ we have the trajectory $\phi(t)=(0, t)^{T}$ and $q^{1}=(0,1)^{T}$. Any formal variation $\phi(t)+\varepsilon \eta(t)$ for control $\varepsilon \mu(t)$ satisfies the linear equation of variations

$$
\dot{\eta}_{1}(t)=\mu(t), \quad \dot{\eta}_{2}(t)=2 \phi_{1}(t) \eta_{1}(t)=0,
$$

with $\eta(0)=\eta(1)=0 ; \mu(0)=\mu(1)=\mu(0)=\dot{\mu}(1)=0$. Hence

$$
\eta_{1}(t)=\int_{0}^{1} \mu(\sigma) d \sigma, \quad \eta_{2}(t)=0 .
$$

Therefore, any formal variation is of the form:

$$
\left(\varepsilon \int_{0}^{t} \mu(\sigma) d \sigma, t\right)^{T}+O\left(\varepsilon^{2}\right) .
$$


We consider the formal variations with order $\varepsilon$ terms only. If these are to be $\varepsilon^{2}$ perturbations of an admissible variation $\xi(\varepsilon, t)$ we should have, for some control $\varepsilon \mu(t)+C^{u}(\varepsilon, t)\left(C^{u}(\varepsilon, t)=O\left(\varepsilon^{2}\right)\right)$

$$
\dot{\xi}_{1}(\varepsilon, t)=\varepsilon \mu(t)+C^{u}(\varepsilon, t), \quad \dot{\xi}_{2}(\varepsilon, t)=\xi_{1}^{2}(\varepsilon, t)+1,
$$

with $\xi(\varepsilon, 0)=q^{0}, \xi(\varepsilon, 1)=q^{1}$. Hence

$$
\begin{aligned}
& \xi_{1}(\varepsilon, t)=\varepsilon \eta_{1}(t)+\int_{0}^{t} C^{u}(\varepsilon, \sigma) d \sigma \stackrel{\nabla}{=} \varepsilon \eta_{1}(t)+C_{1}^{q}(\varepsilon, t), \\
& \xi_{2}(\varepsilon, t)=t+\int_{0}^{t}\left(\varepsilon \eta_{1}(\sigma)+C_{1}^{q}(\varepsilon, \sigma)\right)^{2} d \sigma,
\end{aligned}
$$

with the conditions $\xi_{1}(\varepsilon, 1)=0, \xi_{2}(\varepsilon, 1)=1$, for all $|\varepsilon|$ small. As $\eta_{1}(1)=0$ the first condition is satisfied for all $C_{1}^{q}(\varepsilon, t)$ such that $C_{1}^{q}(\varepsilon, 1)=0$, which can be obtained by an appropriate choice of $C^{u}(\varepsilon, t)$. The second condition implies that

$$
\varepsilon^{2} \int_{0}^{t}\left(\eta_{1}(\sigma)\right)^{2} d \sigma+O\left(\varepsilon^{3}\right)=0 .
$$

So the choice of $C^{u}$ (i.e. $C^{q}$ ) does not influence the $\varepsilon^{2}$ term. Therefore if $\eta_{1}(t)$ is such that $\int_{0}^{t}\left(\eta_{1}(\sigma)\right)^{2} d \sigma \neq 0$, then $\xi_{2}(\varepsilon, 1) \neq 1$. Such a choice can be made. With $\mu(t)=\dot{\eta}_{1}(t)$ we have a formal variation which is no $\varepsilon^{2}$ perturbation of an admissible variation.

The final conclusion of this section is a consequence of Theorem 3.7 and the given formulation of an optimal control problem as a variational problem.

Corollary 5.5. Let OP $\left(\Sigma, \mathscr{G}, q_{0}, h, S\right)$ be a given optimal control problem. Assume that the associated variational problem is normal for a given trajectory-input $\zeta: I \rightarrow B$. Then $\zeta$ is stationary if and only if there exists an injective curve $\eta: I \rightarrow E$ (E the canonical codistribution) such that $\pi_{M} \circ \pi_{E} \circ \eta=\zeta\left(\pi_{M}: M \rightarrow B, \pi_{E}: E \rightarrow M\right.$ natural projections),

$$
\eta_{*}\left(\frac{\partial}{\partial t} \mid{ }_{t}\right) \in \operatorname{ker} d \theta_{\mathscr{G}}
$$

and, in case of a $\mathrm{FE}$ problem,

$$
\left.\left(d \tilde{h}+\theta_{\mathscr{G}}\right)\right|_{\tilde{S}}(\eta(T))=0
$$

where $\theta_{\mathscr{G}}=\pi_{E}^{*}\left(\left.\mathscr{G}\right|_{M} d t\right)+\theta_{E}$ is the Cartan form, $\tilde{h}=\tau \circ \pi_{M} \circ \pi_{E} \circ h$ and $\tilde{S}=$ $\chi((T Q \times\{T\}) \cap M)$ for some section $\chi: M \rightarrow E$.

Formula (5.12) defines a Cartan system. In fact one can use the intrinsic reduction procedure given in [11, § I.e.1.] to study existence and uniqueness of solutions (see [6]).

In many practical optimal control problems $\mathscr{G}$ depends on $q$ and $u$ only. We can work out such a situation in coordinates as we did for the Lagrange problem. Choose natural coordinates $(q, u, \dot{u}, t)$ on $M$ and on $E:(q, u, \dot{u}, \lambda, \mu, t)\left(\beta \in E \Rightarrow \beta=\sum_{i=1}^{n}\right.$ $\lambda_{i} \beta_{i}+\sum_{j+1}^{m} \mu_{j} \beta_{n+j}$ with $\beta_{k}$, cf. (5.6)). Then

$$
\theta_{\mathscr{G}}=\mathscr{G}(q, u) d t+\sum_{i=1}^{n} \lambda_{i}\left(d q_{i}-f_{i}(q, u) d t\right)+\sum_{j=1}^{m} \mu_{j}\left(d u_{j}-\dot{u}_{j} d t\right) .
$$

Some computation shows that condition (5.12) on a stationary curve $\eta(t)=$ 
$(q(t), u(t), \dot{u}(t), \lambda(t), \mu(t), t)$ yields the equations

$$
\begin{aligned}
& \frac{d}{d t} q=f(q, u), \\
& \frac{d}{d t} \lambda=\frac{\partial \mathscr{G}}{\partial q}(q, u)-\left(\frac{\partial f}{\partial q}(q, u)\right)^{T} \lambda, \\
& \frac{\partial \mathscr{G}}{\partial u}(q, u)-\left(\frac{\partial f}{\partial u}(q, u)\right)^{T} \lambda=0, \\
& \mu=0, \quad \frac{d}{d t} u=\dot{u} .
\end{aligned}
$$

With the definition

$$
\mathscr{H}(q, \lambda, u)=\mathscr{G}(q, u)-\lambda^{T} f(q, u)
$$

we obtain the familiar equations of Pontryagin's maximum principle (smooth case):

$$
\dot{q}=-\frac{\partial \mathscr{H}}{\partial \lambda}(q, \lambda, u), \quad \dot{\lambda}=\frac{\partial \mathscr{H}}{\partial q}(q, \lambda, u), \quad \frac{\partial \mathscr{H}}{\partial u}(q, \lambda, u)=0 .
$$

The transversality condition (5.13) yields:

$$
\lambda(T)=\frac{d h}{d q}(q(T))
$$

Remark 5.6. The Lagrange multiplier theorem 3.7 gives a necessary and sufficient condition for formal stationarity. Therefore, these conditions are necessary for optimality. If the problem is not normal, then the conditions of Corollary 5.5 only are sufficient for stationarity. Hence, for nonnormal problems $\phi$ may be stationary (although not necessarily optimal) without being a projection of a stationary $\eta$ in $E$. Higher order theory provides better insight here. That means that we consider $k$ th order conditions:

$$
\left.\frac{d^{j}}{d \varepsilon^{j}}\right|_{\varepsilon=0} \mathscr{I}\left(\phi_{\varepsilon}\right)=0 \quad(j=1, \cdots, k)
$$

and " $k$-formal variations" (variations which satisfy restrictions up to $k$ th order). A Lagrange multiplier theorem similar to 3.7 should then be formulated for " $k$-formal optimality." Obviously we speak of " $k$-normality" in that case and in the CE case $k$ th order local controllability will actually ensure $k$-normality cf. Proposition 5.2 for $k=1$. Other approaches to higher order conditions can be found in the literature. [17] in particular is closely related to the approach suggested here.

Examples of practical optimal control problems to illustrate the given set up would easily lead to complicated calculations in coordinates, which do not essentially differ from the normal approach on $\mathbb{R}^{n}$ except for possible lower dimension and less constraints (e.g. we can use the two-dimensional sphere instead of $\mathbb{R}^{3}$ with a restriction to the sphere). We did not search for examples where the coordinate-free approach might be profitable. Some may be found in [11]. The given approach can be generalized to the infinite horizon optimal control problem (see [6]). We expect that our approach might be particularly profitable there, for instance to obtain methods for computation of optimal feedback controls in infinite horizon problems.

Acknowledgments. Special gratitude is owed to $\mathrm{H}$. Nijmeijer for numerous discussions about the subject of this paper. Furthermore, I would like to thank 
Prof. J. C. Willems for introducing me to this research area and Dr. J. H. van Schuppen, Prof. F. Takens and an anonymous referee for valuable suggestions.

\section{REFERENCES}

[1] V. I. ARNOLD (1973), Ordinary Differential Equations, MIT Press, Cambridge, MA.

[2] R. M. BIANCHINI AND G. STEFANI (1982), Local controllability for analytic families of vector fields, Rep. Universita' degli Studi di Firenze.

[3] G. A. BLIss (1946), Lectures on the Calculus of Variations, Univ. Chicago Press, Chicago.

[4] R. W. BRocketr (1977); Control theory and analytical mechanics, in 1976 Ames Research Center Conference on Geometric Control Theory, C. Martin and R. Hermann, eds., Math. Sci. Press.

[5] - (1976), Nonlinear systems and differential geometry, Proc. IEEE, 64, pp. 61-72.

[6] J. C. P. Bus (1983), Infinite horizon optimal control on manifolds, Paper presented at Berkeley-Ames Conference on Nonlinear Problems in Control and Fluid Dynamics.

[7] E. CARTAN (1922), Leçons sur les invariants intégraux, Hermann, Paris.

[8] C. CARATHÉODORY (1935), Variationsrechnung und partielle Differentialgleichungen erster Ordnung, Teubner, Leipzig.

[9] P. CROUCH (1981). Dynamical realizations of finite Volterra series, this Journal, 19, pp. 177-202.

[10] R. B. GARDNER (1983), Differential geometric methods interfacing control theory, in Differential Geometric Control Theory, R. W. Brockett, R. S. Millman and H. J. Sussman, eds., Birkhäuser, Boston.

[11] P. A. GRIFfiths (1983), Exterior Differential Systems and the Calculus of Variations, Birkhäuser, Boston.

[12] R. HERMANN (1962), Some differential-geometric aspects of the Lagrange variational problem, Illinois J. Math., 6, pp. 634-673.

[13] - (1977), Differential Geometry and the Calculus of Variations, 2nd ed., Interdisc. Math. XVII, Math. Sci. Press.,

[14] R. HERMANN AND A. KRENER (1977), Nonlinear controllability and observability, IEEE Trans. Automat. Control, AC-22, pp. 728-740.

[15] H. HeRMES (1974), On local and global controllability, this Journal, 12, pp. 252-261.

[16] - (1982), On local controllability, this Journal, 20, pp. 211-220.

[17] A. J. KRENER (1977), The high order maximal principle and its application to singular extremals, this Journal, 15, pp. 256-293.

[18] H. NIJMEIJER AND A. VAN DER SCHAFT (1982), Controlled invariance for nonlinear systems, IEEE Trans. Automat. Control. AC-27, pp. 904-914.

[19] M. SpIVAK (1979), A Comprehensive Introduction to Differential Geometry, Vols. I and II, Publish or Perish Inc., Berkeley, CA.

[20] F. TAKENS (1978), Unpublished course notes.

[21] J. C. Willems (1979), System theoretic models for the analysis of physical systems, Ric. di Autom., Spec. Issue on Systems theory and Physics.

[22] L. C. Young (1969), Lectures on the Calculus of Variations and Optimal Control Theory, W. B. Saunders, Philadelphia. 